\title{
Transdifferentiation of Small Cell Carcinoma of the Urinary Bladder from Urothelial Carcinoma after Transurethral Resection of a Bladder Tumor, Intravesical Bacillus Calmette-Guerin Instillation, and Chemotherapy: A Case Report
}

\author{
Kento Morozumi ${ }^{\mathrm{a}}$ Shunichi Namiki ${ }^{\mathrm{a}}$ Takashi Kudo $^{\mathrm{a}}$ Masataka Aizawa ${ }^{\mathrm{a}}$ \\ Naomasa Ioritani ${ }^{a}$ Kazuhiro Sakamoto ${ }^{b}$ Yasuhiro Nakamura ${ }^{c}$ \\ aDepartment of Urology, Japan Community Health Care Organization Sendai Hospital, \\ Sendai, Japan; ${ }^{b}$ Department of Pathology, Osaki Citizen Hospital, Osaki, Japan; ${ }^{\mathrm{C}}$ Division \\ of Pathology, Faculty of Medicine, Tohoku Medical and Pharmaceutical University, \\ Sendai, Japan
}

\section{Keywords}

Transdifferentiation · Small cell carcinoma · Urinary bladder · Bacillus Calmette-Guerin . Chemotherapy

\begin{abstract}
A 73-year-old male underwent transurethral resection of a bladder tumor in August 2010 and April 2011. Pathological examination revealed urothelial carcinoma. After the surgery, chemotherapy and intravesical Bacillus Calmette-Guerin instillation were performed. In September 2014, he once again underwent transurethral resection of the bladder tumor for recurrence, and was again diagnosed with urothelial carcinoma, pT2, by pathological examination. After neoadjuvant chemotherapy, radical cystectomy for tumor recurrence was performed. Pathological examination at this time revealed small cell carcinoma, pT3N0. It is rare
\end{abstract}


for urothelial carcinoma to change to small cell carcinoma, and the mechanism and cause of this change are still unknown. In this case report, we discuss what causes small cell carcinoma of the urinary bladder and review the literature regarding its origin.

(C) 2016 The Author(s)

Published by S. Karger AG, Basel

\section{Introduction}

Small cell carcinoma (SCC) of the urinary bladder is rarer and has a worse prognosis compared to urothelial carcinoma (UC), and accounts for less than $1 \%$ of all primary bladder carcinomas [1]. Since the first reported case published in 1981 by Cramer et al. [2], SCC of the urinary bladder has been described in many case reports. It is even rarer for UC to change to SCC, and there has been no case report of the change after intravesical Bacillus Calmette-Guerin (BCG) instillation and chemotherapy in the English literature.

Recently, in the department of pathology, transdifferentiation has been receiving a lot of attention. In this report, we present a case of transdifferentiation of UC to SCC after treatment with intravesical BCG instillation and chemotherapy, and we review the literature and discuss the mechanism of the transdifferentiation.

\section{Case Report}

A 73-year-old man underwent transurethral resection of a bladder tumor (TURBT) for the first time in August 2010, at which time pathological examination of the surgical specimens revealed UC, pT1, G2. After TURBT, intra-arterial injection chemotherapy with epirubicin/cisplatin and intravesical BCG instillation were performed. In April 2011, he underwent a second TURBT for bladder tumor recurrence. As pathological examination again revealed UC, pTis, G3, intravesical BCG instillation was performed again.

In September 2014, he underwent a third TURBT for bladder tumor recurrence on the right side of the bladder wall. Since pathological examination revealed UC, pT2, neoadjuvant chemotherapy with 2 courses of gemcitabine/cisplatin was performed beginning in October 2014. As a fourth TURBT and full-body CT scan revealed no more evidence of tumor in January 2015, he was closely followed up by cystoscopy and CT scan. However, in March 2015, cystoscopy revealed bladder tumor recurrence on the right side wall, and SCC was suspected by urine cytology. His serum neuron-specific enolase (NSE) level was $7.7 \mathrm{ng} / \mathrm{mL}$ (reference values $10.0 \mathrm{ng} / \mathrm{mL}$ ). A full-body CT scan showed increased thickness of the urinary bladder wall on the right side with right hydronephrosis, but no obvious metastasis (Fig. 1). Hence, he underwent radical cystectomy and lymphadenectomy with construction of an ileal conduit in April 2015. His postoperative course was uneventful and serum NSE was $8.9 \mathrm{ng} / \mathrm{mL}$ before discharge, which was within reference range. Pathological examination revealed SCC with squamous cell carcinoma, pT3N0 (Fig. 2a). There was no UC component. Thorough overview of all the series of specimens revealed that in the third TURBT specimen, UC made up the majority of the tumor, with a very small component of SCC that stained positive during synaptophysin immunostaining (Fig. 2b). Ki67 counts increased over time (48\% in the first TURBT specimen in 2010, 53\% in the third TURBT specimen in 2014 and $90 \%$ in the cystectomy specimen in 2015). From these histopathological findings, it became clear that the bladder cancer had already changed to SCC at the time of the third TURBT. 


\section{Case Reports in Oncology}

Adjuvant chemotherapy was not performed. In June 2015, he noticed bilateral edema of the lower extremities. Serum NSE showed an increase to $262 \mathrm{ng} / \mathrm{mL}$, and PET-CT scan showed pelvic lymph node metastases and peritoneal dissemination. He underwent chemotherapy with etoposide/carboplatin commencing in August 2015. The metastatic lesions demonstrated a partial response during the first 4 courses, but a full-body CT scan at the end of the fifth course showed new liver metastasis. The chemotherapeutic agent was changed to amrubicin from January 2016. However, after the first course of amrubicin, the patient developed intestinal perforation due to necrosis secondary to peritoneal dissemination, and he passed away in February 2016.

\section{Discussion}

In our case, pathology examination of the first and second TURBT specimens indicated UC, but the tumor changed to UC with a small component of SCC by the third TURBT. During this period, intra-arterial injection chemotherapy with epirubicin/cisplatin and intravesical BCG instillation were performed. Despite this, the radical cystectomy specimen after chemotherapy with gemcitabine/cisplatin changed to SCC without UC. Ours is one of very few reports regarding the origin of SCC of the urinary bladder. Eight SCC of the urinary bladder cases, including ours, have been reported to have a past history of UC [3]. The reported time to occurrence of SCC after the first TURBT is from 24 to 164 months (median time 70 months). Intravesical BCG instillation was performed in 7 of the reported cases, and only 4 patients received chemotherapy. Five of the cases showed evidence of SCC only in the resection specimen, and not in the TURBT specimens. Three cases with adjuvant chemotherapy had a good prognosis.

There are several hypotheses in the literature explaining the origin of SCC of the urinary bladder [4]. According to the first theory, decarboxylation cells that originate from the neural crest migrate to the urinary bladder, and SCC occurs by canceration of these cells. Decarboxylation cells present intracytoplasmic neurosecretory granules and can be positively stained with chromogranin A. A second theory suggests that SCC arises from neuroendocrine stem cells that exist in the mucosa of the urinary bladder. According to a third theory, SCC originates from multipotent cells that can be converted to different tissue types. This third theory is believed to be the most plausible, as tumor specimens of SCC of the urinary bladder often include several other histological components, such as UC, adenocarcinoma, and squamous cell carcinoma. Contradictory to this theory, in our case, despite the fact that there were only UC components in the first and second TURBT specimens, SCC appeared newly in the third TURBT, and all UC components changed to SCC in the cystectomy specimen.

There are 2 possible mechanisms for the change to SCC in our case. The first possibility is that the UC component was completely treated by the therapies administered, with a simultaneous relative increase in the SCC component. The other is that UC transdifferentiated to SCC with these therapies. Although it is difficult to determine the exact pathological process in our case, we believe that transdifferentiation by these therapies is most likely. The first reason is that histopathological findings changed by the chemotherapy and intravesical BCG instillation. UC with SCC in the third TURBT specimen and SCC with squamous cell carcinoma in the cystectomy specimens appeared newly, while only the UC component was evident in the first and second TURBT specimen. In other words, different histopathological components appeared newly after these therapies. The second reason is that Ki67 counts increased with histopathological change. As Ki67 counts indicate the direct correlation between its 
expression and malignancy of the tumor, we examined Ki67 counts and evaluated the malignancy. In urological practice, we often encounter transdifferentiation of prostate cancer to neuroendocrine cancer after hormone therapy and chemotherapy [5]. In the field of gastroenterology, cases of transdifferentiation after use of platinum antitumor agents have been reported [6]. However, no reports exist regarding transdifferentiation of UC to SCC following intravesical BCG instillation; most SCC of the urinary bladder cases which had a past history of UC underwent intravesical BCG instillation. In addition, since it has been reported that the form change of bladder epithelium lasts for a long time by intravesical BCG instillation [7], chemotherapy and BCG may lead to transdifferentiation.

High-risk nonmuscle invasive bladder cancer (NMIBC) is reported to have 5-year recurrence rates as high as $80 \%$ and progression rates up to $45 \%$. European Association of Urology guidelines recommend that high-risk NMIBC should be treated with intravesical BCG instillation for bladder preservation [7]. However, BCG refractory cases are not suitable for bladder preservation, and cases of recurrence after second-line BCG therapy have a poor prognosis. So far, a standard treatment strategy for SCC of the urinary bladder has not yet been established. Recently, the efficacy of adjuvant or neo-adjuvant chemotherapy similar to chemotherapy regimens for SCC of lung cancer, together with radical cystectomy or radiation, has been reported [8-10]. This indicates that an accurate diagnosis of SCC of the urinary bladder is related to better prognosis. In BCG-refractory UC cases, as in our case, the existence of SCC should be considered, and multimodal therapy should be administered as early as possible.

\section{Statement of Ethics}

The authors have no ethical conflicts to disclose.

\section{Disclosure Statement}

The authors declare no conflict of interest in association with this article.

\section{References}

1 Prelaj A, Rebuzzi SE, Magliocca FM, Speranza I, Corongiu E, Borgoni G, Perugia G, Liberti M, Bianco V: Neoadjuvant chemotherapy in neuroendocrine bladder cancer: a case report. Am J Case Rep 2016;17:248-253.

-2 Cramer SF, Aikawa M, Cebelin M: Neurosecretory granules in small cell invasive carcinoma of the urinary bladder. Cancer 1981;47:724-730.

3 Goto K, Hayashi T, Inoue Y, Hayashi M: Small cell carcinoma occurring after treatment for urothelial cell carcinoma of the urinary bladder: a case report. Nishinihon J Urol 2010;72:697-700.

4 Celik O, Ekin G, Ipekci T, Budak S, Ilbey YO: Diagnosis and treatment in primary bladder small cell carcinoma: literature review. Arch Ital Urol Androl 2016;88:52-55.

5 Cerasuolo M, Paris D, Iannotti F, Melck D, Verde R, Mazzarella E, Motta A, Ligresti A: Neuroendocrine transdifferentiation in human prostate cancer cells: an integrated approach. Cancer Res 2015;75:29752986.

-6 Hiroshi N, Genshu T, Takahiro U: A case of esophageal squamous cell carcinoma with an adenocarcinoma component that dedifferentiated after chemotherapy. Jpn J Cancer Chemother 2014;41:909-912.

-7 Babjuk M, Burger M, Zigeuner R, Shariat S-F, van Rhijn B-W, Comperat E, Sylvester R-J, Kaasinen E, Bohle A, Palou Redorta J, Roupret M: EAU guidelines on non-muscle-invasive urothelial carcinoma of the bladder: update 2013. Eur Urol 2013;6:639-653. 


\section{Case Reports in Oncology}

Morozumi et al: Transdifferentiation of Small Cell Carcinoma of the Urinary Bladder from Urothelial Carcinoma after Transurethral Resection of a Bladder Tumor, Intravesical Bacillus Calmette-Guerin Instillation, and Chemotherapy: A Case Report

-8 Lynch SP, Shen Y, Kamat A, Grossman H-B, Shah J-B, Millikan R-E, Dinney C-P, Siefker-Radtke A: Neoadjuvant chemotherapy in small cell urothelial cancer improves pathologic downstaging and longterm outcomes: results from a retrospective study at the MD Anderson Cancer Center. Eur Urol 2013;64:307-313.

-9 Kaushik D, Frank I, Boorjian SA, Cheville JC, Eisenberg MS, Thapa P: Long-term results of radical cystectomy and role of adjuvant chemotherapy for small cell carcinoma of the bladder. Int J Urol 2015;22:549-554.

10 Pasquier D, Barney B, Sundar S, Poortmans P, Villa S: Small cell carcinoma of the urinary bladder: a retrospective, multicenter rare cancer network study of 107 patients. Int J Radiat Oncol Biol Phys 2015;92:904-910.

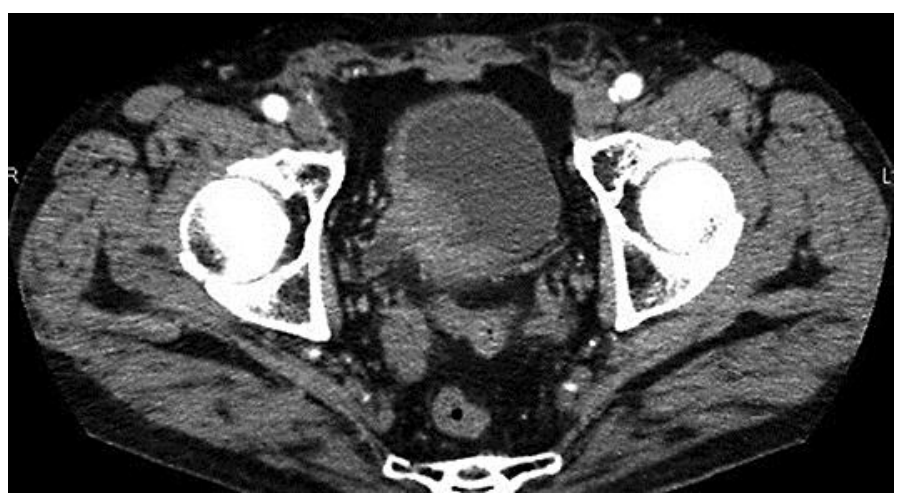

Fig. 1. A full-body CT scan in March 2015 showing a further increase in thickness of the urinary bladder wall on the right side, with right hydronephrosis and no metastasis. 


\section{Case Reports in Oncology}

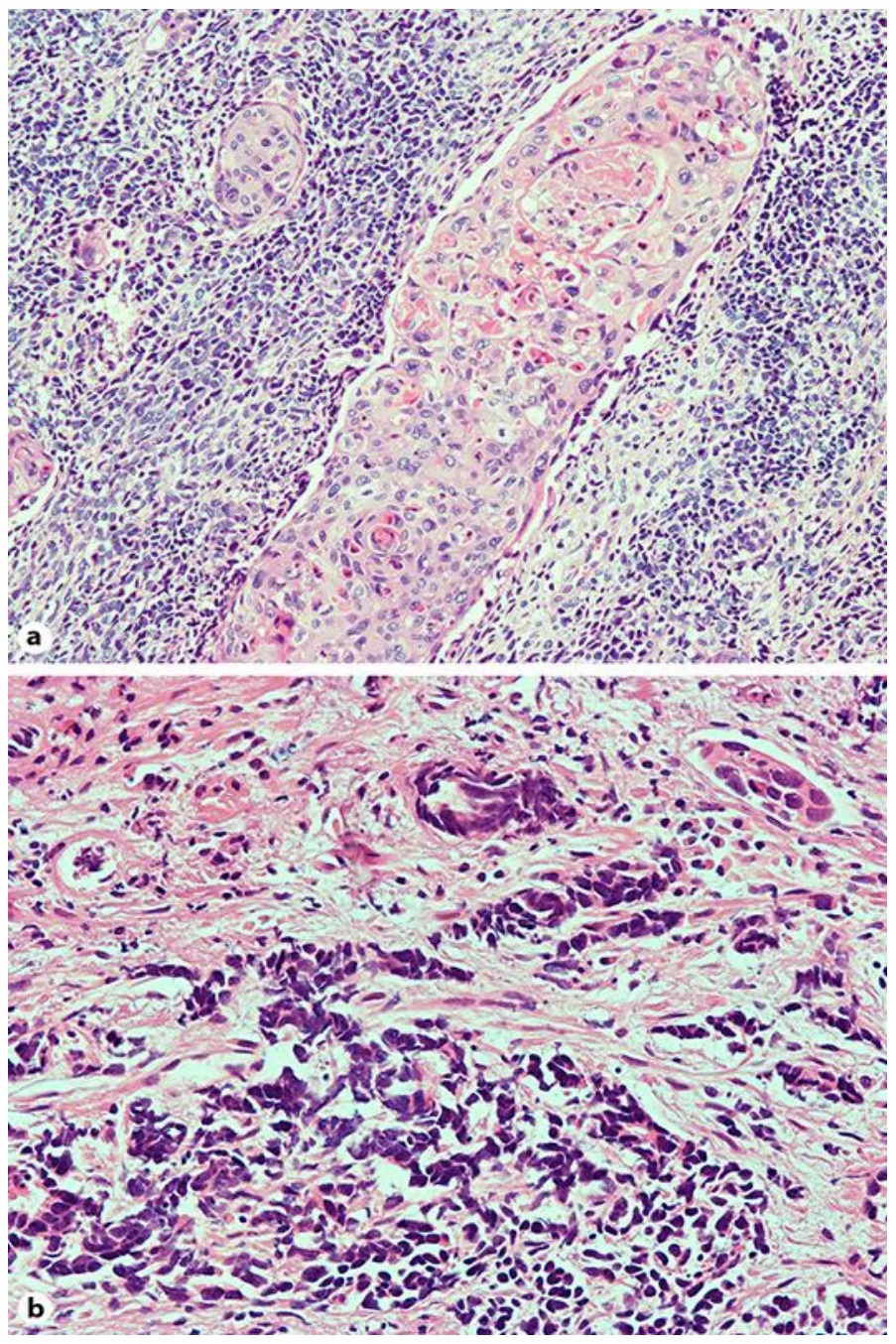

Fig. 2. a Pathological examination of the radical cystectomy specimen in April 2015 revealing small cell carcinoma with squamous cell carcinoma. HE. $\times 10$. b Pathological examination of the third transurethral resection of bladder tumor specimen in September 2014 revealing that urothelial carcinoma constituted a majority of the tumor, with a small component of small cell carcinoma. HE. $\times 20$. 